\title{
Motivational Interviewing als Kurzintervention bei Menschen mit Alkoholproblemen: Georg Kremer Stand der Forschung und Praxis
}

\author{
Brief Interventions Adapted from Motivational Interviewing for People with \\ Alcohol Problems: Current Evidence and Review of Applications in Germany
}

\section{Zusammenfassung}

Der vorliegende Beitrag fasst die Studienlage zu Kurzinterventionen auf der Basis des Motivational Interviewing (MI) bei alkoholbezogenen Störungen zusammen. Als Datengrundlage dienen aktuelle Metaanalysen zu Kurzinterventionen sowie ergänzende aktuelle MI-Studien. Die Ergebnisse belegen eine hinreichende Evidenz von MI-Kurzinterventionen im Vergleich zu Kontrollgruppen, die keine Intervention erhalten haben. Dies gilt vor allem für Menschen mit leichten bis mittelgradigen alkoholbezogenen Problemen. Die Effektstärken liegen im unteren bis mittleren Bereich. Spezifische Wirkfaktoren werden diskutiert. Die praktische Umsetzung von MI-Kurzinterventionen in Deutschland befindet sich in den Anfängen. Außerhalb der traditionellen Suchtkrankenhilfe wurden verschiedene Modellvorhaben und Weiterbildungscurricula unter Einbezug des MIKurzinterventionskonzepts entwickelt und erprobt.

\section{Schliuisselwörter}

Kurzinterventionen · Motivational Interviewing · alkoholbezogene Störungen · Effektivität · Implementierung

\section{Abstract}

This paper summarizes the current evidence on MI-based brief interventions for people with alcohol problems. Data are used from two current meta-analyses published in 2001 and four additional randomized controlled trials mostly published in the following years. There is a substantial evidence for MI-based brief interventions when compared to controls. Results are best for people with mild to moderate alcohol problems, but even for people with substantial or severe alcohol problems MI-based brief interventions lead to a higher specialized treatment attendance rate. Effect sizes are on the small to medium range.

The question of specific effective elements is discussed. It seems to be that rather unspecific elements like empathy, feedback, responsiblity and non-aggressive counselling style than raising specific substance use or lifestyle topics are responsible for the effectiveness of interventions.

Current applications of MI-based brief interventions in Germany are described. There are some encouraging experiences in the primary health care and general hospital but due to a lack of financial support there is no transfer in routine care. Three curricula for the training of non-specialists in dealing with substance use problems are described. Main part of all curricula is information about MI-based brief interventions and appropriate practical exercises.

Key words

Brief interventions $\cdot$ motivational interviewing $\cdot$ alcohol problems · effectiveness · implementation 
Einführung: Kurzinterventionen bei alkoholbezogenen Störungen

Der Begriff Kurzintervention (KI) wird häufig nicht ausreichend definiert oder von anderen Begriffen (Früherkennung, Frühintervention, Motivationsbehandlung, Sekundärprävention, schadensmindernde Strategien etc.) abgegrenzt. In der Literatur werden darüber hinaus sehr unterschiedliche Interventionsansätze und -konzepte als „Kurzintervention“ bezeichnet [1]. Sie lassen sich unter vier Aspekten voneinander differenzieren:

1) Inhalt. Kurzinterventionen basieren weitgehend auf zwei theoretischen Ansätzen:

Motivational Interviewing (MI) [2, 3], für die medizinische Versorgung von Rollnick et al. [4] als „Brief Motivational Interviewing“ adaptiert. Dieser KI-Ansatz betont insbesondere die motivationalen Aspekte einer möglichen Verhaltensänderung und berücksichtigt neben den Grundprinzipien des MI folgende Themen:

- Lebensführung, Stressoren und Substanzgebrauch

- Gesundheit und Substanzgebrauch

- $\quad$ ein typischer Tag

- die guten und die weniger guten Dinge des

Substanzgebrauchs

Informationen geben

Zukunft und Gegenwart

Sorgen und Beunruhigungen herausarbeiten

Hilfe bei der Entscheidungsfindung

Kognitiv-behaviorale Verfahren, hier insbesondere das SelbstKontroll-Konzept, wie es von Hester \& Miller [5] entwickelt und von Heather et al. [6] als „Skills Based Counselling“ für die medizinische Versorgung adaptiert wurde. Dieser KI-Ansatz betont die pragmatischen Aspekte einer Verhaltensänderung und berücksichtigt folgende Themen:

- $\quad$ Trinkverhalten und Lebensführung

- Vergleiche zwischen individuellem Konsum und „normalem“ Konsum

- Informationen über zu empfehlende Trinkmengen

- Instruktionen zur Selbstbeobachtung

- $\quad$ Tipps zur Verringerung der Häufigkeit des Trinkens

- Identifikation von Hoch-Risiko-Situationen

- Instruktionen, wie mit diesen Situationen umzugehen ist, ohne zu trinken

- Diskussion über alternative Verhaltensweisen im Rahmen einer veränderten Lebensführung.

Unter dem Akronym FRAMES werden von Miller \& Sanchez [7] sechs wesentliche Elemente von KI beschrieben, die sich als wirksam erwiesen haben:

- persönliches Feedback geben (Feedback)

- die persönliche Verantwortung des Betroffenen betonen (Responsibility)

- $\quad$ einen konkreten Ratschlag geben (Advice)

- $\quad$ eine Reihe alternativer Veränderungsstrategien vorschlagen und diskutieren (Menus)

- Empathie ausdrücken (Empathy)

- die Selbstwirksamkeit stärken (Self-efficacy).
2) Länge. Babor [8, zit. nach 1] unterscheidet „minimal“ (einmalige Intervention von bis zu fünf Minuten), „brief“ (maximal drei Sitzungen von jeweils bis zu 60 Minuten), „moderate“ (fünf bis sieben Sitzungen) und „intensive interventions“ (acht oder mehr Sitzungen). In der deutschsprachigen Literatur werden „minimal“ und „brief interventions“ im Sinne Babors in der Regel zusammenfassend als KI bezeichnet.

3) Behandlungskonzept. Es muss unterschieden werden zwischen Kurzinterventionen, die als einzige suchtmittelspezifische Intervention angeboten werden, und Kurzinterventionen, die als Ergänzung zu umfassenderen Behandlungsprogrammen eingesetzt werden.

4) Versorgungspolitischer Kontext. Kurzinterventionen sind zumeist frühe Interventionen - Veränderungsbereitschaft bzw. Behandlungsmotivation sollen vor Eintritt (etwa bereits bei riskantem oder schädlichem Gebrauch) bzw. in frühen Phasen der Abhängigkeitsentwicklung gefördert werden - und wurden zumeist im Rahmen der medizinischen Basisversorgung evaluiert, seltener im Rahmen suchtspezifischer oder bevölkerungsbezogener Kontexte. Auch muss unterschieden werden zwischen ambulant und stationär durchgeführten KI.

Zusammengefasst lässt sich festhalten, dass die in der Literatur beschriebenen KI sich in erheblichem Ausmaß hinsichtlich Inhalt, Dauer, Kontext, Vorgehen sowie einer Reihe weiterer Merkmale unterscheiden. Generalisierende Schlussfolgerungen sind daher stets sorgfältig zu prüfen.

Eine aktuelle Metaanalyse zu KI bei Alkoholproblemen berücksichtigt 54 Studien, die in den Jahren 1970-1998 als randomisierte und kontrollierte Studien veröffentlicht worden sind [9]. KI werden als ein- oder mehrmalige (bis zu vier) Interventionen von jeweils 5-60 Minuten Dauer definiert. Sie sind sämtlich als Alternative zu keiner oder einer intensiveren Behandlung konzipiert. Die Autoren schlussfolgern:

- $\quad$ Es liegt eine hinreichende Evidenz für die Wirksamkeit von KI im Vergleich zu Kontrollgruppen vor, die keine Intervention erhalten haben.

- Dies gilt vor allem für Menschen mit leichten bis mittelgradigen alkoholbezogenen Problemen.

- Die Effektstärken liegen im unteren bis mittleren Bereich.

Küfner [10] kommt in seiner Übersichtsarbeit zu folgenden Ergebnissen:

- $\quad$ Die Wirksamkeit von KI und Kurztherapien ist generell sehr gut nachgewiesen.

- $\quad$ KI im Sinne einer minimalen Behandlung bis drei oder vier Sitzungen eignen sich gut für Änderungen des Risikokonsums von Alkohol.

Mehrere Studien, die in Deutschland in der medizinischen Versorgung durchgeführt wurden, zeigten darüber hinaus, dass KI zu einer deutlichen Steigerung der Inanspruchnahme weiterführender Hilfen (z.B. Suchtberatungsstellen) bei Alkoholabhängigen führten [11 -13]. Es konnte gezeigt werden, dass der KI-Ansatz viele Patienten erreicht, die bisher das suchtspezifische Hilfesystem noch nicht in Anspruch genommen haben, was den 
sekundärpräventiven Aspekt von KI-Konzepten im Alkoholbereich unterstreicht.

\section{Motivational Interviewing (MI) als Kurzintervention: Stand der Forschung}

Dunn et al. [14] berücksichtigten 29 Studien zu KI auf Basis des MI mit unterschiedlichen Problemlagen in unterschiedlichen Settings, davon 17 Studien im Rahmen der ambulanten oder stationären Behandlung substanzbezogener Störungen, 9 davon bei alkoholbezogenen Störungen mit einem mindestens zweimonatigen Katamnesezeitraum (vgl. Tab.1). Unter diesen neun Studien wurden drei im Allgemeinkrankenhaus oder in allgemeinen psychosozialen Beratungsstellen, zwei in fachspezifischen Einrichtungen (analog unseren Suchtberatungsstellen) und eine in einer medizinischen Ambulanz durchgeführt. Sechs Studien untersuchten hauptsächlich die Effekte des MI gegenüber einer anderen Behandlungsform, zwei Studien untersuchten die Effekte von MI allein gegenüber einer Kontrollbedingung (Warteliste), eine Studie ergänzte eine Standardbehandlung durch MI-Komponenten und verglich dies mit der Standardbehandlung allein (teilweise mehrere Hypothesentestungen). Die Länge der Interventionssitzungen betrug zwischen 30 und 60 Minuten, in zwei Studien kam es zu mehreren (zwei bzw. vier) Sitzungen.

Es konnte in der Summe aller Studien eine eindeutige positive Evidenz mit Effektstärken im unteren bis mittleren Bereich für KI auf Basis des MI nachgewiesen werden. Sieben der neun Studien wiesen, bezogen auf die Hauptergebniskriterien zu allen Erhebungszeitpunkten, positive Effekte auf. Lediglich eine Studie (Project MATCH [17]) wies zu beiden Erhebungszeitpunkten gegenüber den anderen Interventionsarmen (TSF: 12 Schritte der Anonymen Alkoholiker, CBT: kognitiv-behavioraler Beratungsansatz) geringfügig negative Evidenzstärken auf.
Im Hinblick auf differenzierte Indikationen zu eher motivationalen oder eher verhaltensbezogenen KI konnten Heather et al. [6] zeigen, dass Patienten, die vor Beginn der KI noch nicht entschieden waren, ihr Konsumverhalten zu ändern, von einem motivationalen Ansatz im Sinne des MI signifikant häufiger profitierten.

Vier weitere Studien, die von Dunn et al. nicht berücksichtigt wurden (vgl. Miller [23]), sollen im Folgenden kurz beschrieben werden (vgl. Tab. 2). Alle Studien wurden in Settings der medizinischen Versorgung durchgeführt.

Senft et al. [24] führten in Portland (USA) eine Studie in der ambulanten medizinischen Versorgung durch. 516 riskant konsumierende Patienten (,heavy drinkers“) wurden nach einem Routinescreening mit dem AUDIT [28] randomisiert einer Kontroll- und einer Interventionsbedingung zugeteilt. Die Kontrollgruppe erhielt die übliche ärztliche Standardbehandlung. Die Intervention bestand in einem kurzen ärztlichen Ratschlag, einem anschließenden ca. 15-minütigen Gespräch auf der Basis des MI sowie Informationsmaterialien. Nach 6 und nach 12 Monaten konnten 392 bzw. 336 Patienten erneut zu ihren Alkoholkonsumgewohnheiten befragt werden. Nach 6 Monaten wiesen die Patienten der Interventionsgruppe signifikant weniger Trinktage und Trinkmengen auf als die Patienten der Kontrollgruppe $(\mathrm{p}<.02$ bzw. $\mathrm{p}<.04)$, nach 12 Monaten war allerdings nur noch der Unterschied in den Trinktagen signifikant $(\mathrm{p}<.04)$, der Unterschied in den Gesamttrinkmengen nicht mehr signifikant ( $\mathrm{p}<.13$ ). Dies veranlasste die Autoren zu der Schlussfolgerung, dass einmalige kurze Interventionen zwar effektiv, aber auf die Dauer nicht ausreichend seien. Das Potenzial der ambulanten medizinischen Versorgung bestünde allerdings gerade darin, dass die Patienten hier in aller Regel mehrmals im Jahr gesehen würden und gezielte Interventionen wiederholt bzw. weitergeführt werden könnten.

Tab. 1 Studien zu MI-Kurzinterventionen bei alkoholbezogenen Störungen nach Dunn et al. 2001

\begin{tabular}{|c|c|c|c|c|c|c|c|}
\hline Erstautor und Jahr & $n$ & Setting & Haupttestung & $\begin{array}{l}\text { Dauer der Inter- } \\
\text { vention (min.) }\end{array}$ & $\begin{array}{l}\text { Hauptergebnis- } \\
\text { kriterium }\end{array}$ & $\begin{array}{l}\text { Nachbefra- } \\
\text { gungszeitraum }\end{array}$ & Effektstärken \\
\hline Bien 1993 [15] & 31 & „Sucht“-Beratungsstelle & $\begin{array}{l}\text { MI + Standard vs. } \\
\text { Standard }\end{array}$ & 60 & Drinks pro Woche & $\begin{array}{l}3 \text { Monate } \\
6 \text { Monate }\end{array}$ & $\begin{array}{l}0,72 \\
0,35\end{array}$ \\
\hline Miller 1993 [16] & 42 & andere psychosoziale Beratung & $\begin{array}{l}\text { MI vs. andere } \\
\text { Intervention }\end{array}$ & 60 & Drinks pro Woche & 12 Monate & 0,78 \\
\hline Heather 1996 [6] & 174 & Allgemeinkrankenhaus & $\begin{array}{l}\text { MI vs. andere } \\
\text { Intervention }\end{array}$ & 35 & Drinks pro Woche & 6 Monate & 0,35 \\
\hline $\begin{array}{l}\text { MATCH } 1997 \\
\text { (ambulant) [17] }\end{array}$ & 1726 & „Sucht“-Beratungsstelle & $\begin{array}{l}\text { MI vs. andere } \\
\text { Interventionen }\end{array}$ & $(4 \times 60) 240$ & $\begin{array}{l}\text { negative Konse- } \\
\text { quenzen }\end{array}$ & $\begin{array}{l}9 \text { Monate } \\
15 \text { Monate }\end{array}$ & $\begin{array}{l}\text { Vs. CBT: }-0,09 \\
\text { Vs. TSF: }-0,30 \\
\text { Vs. CBT: }-0,01 \\
\text { Vs. TSF: }-0,18\end{array}$ \\
\hline Marlatt 1998 [18] & 348 & andere psychosoziale Beratung & $\begin{array}{l}\text { MI vs. keine } \\
\text { Behandlung }\end{array}$ & 60 & $\begin{array}{l}\text { alkoholbezogene } \\
\text { Probleme }\end{array}$ & $\begin{array}{l}12 \text { Monate } \\
24 \text { Monate }\end{array}$ & $\begin{array}{l}0,35 \\
0,35\end{array}$ \\
\hline Handmaker 1999 [19] & 42 & Allgemein-Krankenhaus & $\begin{array}{l}\text { MI vs. andere } \\
\text { Intervention }\end{array}$ & 60 & abstinente Tage & 2 Monate & 0,38 \\
\hline Monti 1999 [20] & 94 & Allgemein-Krankenhaus & $\begin{array}{l}\text { MI vs. andere } \\
\text { Intervention }\end{array}$ & 35 & $\begin{array}{l}\text { alkoholbezogene } \\
\text { Probleme }\end{array}$ & 6 Monate & 0,45 \\
\hline Gentilello 1999 [21] & 762 & Allgemein-Krankenhaus & $\begin{array}{l}\text { MI vs. keine } \\
\text { Behandlung }\end{array}$ & 30 & Drinks pro Woche & $\begin{array}{l}6 \text { Monate } \\
12 \text { Monate }\end{array}$ & $\begin{array}{l}-0,08 \\
0,09\end{array}$ \\
\hline Schneider 1999 [22] & 89 & andere psychosoziale Beratung & $\begin{array}{l}\text { MI vs. andere } \\
\text { Intervention }\end{array}$ & $(2 \times 60) 120$ & $\begin{array}{l}\text { Addiction Severity } \\
\text { Index (ASI) }\end{array}$ & $\begin{array}{l}3 \text { Monate } \\
9 \text { Monate }\end{array}$ & $\begin{array}{l}0,24 \\
0,42\end{array}$ \\
\hline
\end{tabular}


Tab. 2 Weitere Studien zu MI-Kurzinterventionen bei alkoholbezogenen Störungen

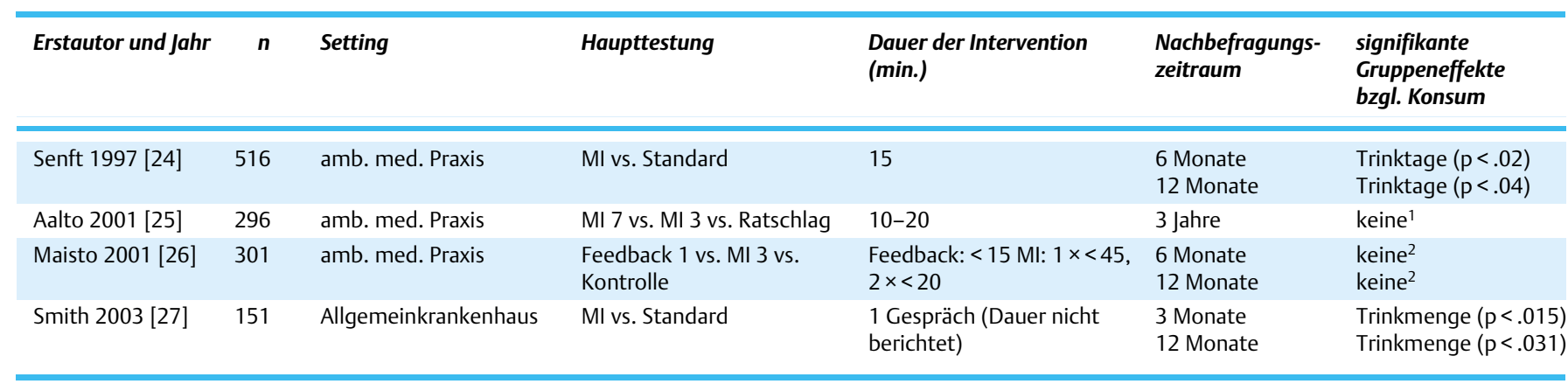

${ }^{1}$ Besserung des MCV-Werts ( $\left.\mathrm{p}<.01\right)$, CDT tendenzielle Verringerung und Konsumreduktion über alle Gruppen

${ }^{2}$ deutliche Konsumreduktion über alle Gruppen

Aalto et al. [25] führten in Lahti (Finnland) ebenfalls in der ambulanten medizinischen Versorgung eine KI-Studie mit nicht abhängigen sog. „heavy drinkers“ durch. 296 Patienten wurden nach einem positiven Screening im CAGE ([29], cut-off 3) oder bei einem berichteten wöchentlichen Alkoholkonsum über $280 \mathrm{~g}$ (ca. 35 Einheiten) randomisiert drei Studienbedingungen zugeteilt: Die Interventionsgruppe A erhielt innerhalb eines Zeitraums von $2^{1} / 2$ Jahren $7 \mathrm{KI}$, die Interventionsgruppe B innerhalb desselben Zeitraums 3 KI, die Kontrollgruppe C erhielt zu Beginn des Beobachtungszeitraums lediglich einen kurzen Ratschlag des Arztes, den Alkoholkonsum zu reduzieren. Die Interventionen basierten auf den FRAMES (s.o.) und wurden bis auf wenige Ausnahmen vom behandelnden niedergelassenen Arzt im Rahmen des Routinesettings der Praxis durchgeführt. Nach drei Jahren ergaben sich weder über die gesamte untersuchte Gruppe noch zwischen Interventions- und Kontrollgruppe signifikante Veränderungen der Konsumgewohnheiten. Es wurde zwar über alle Gruppen weniger getrunken als zu Beginn der Untersuchung, jedoch einzig der MCV-Wert verbesserte sich über die Gesamtgruppe signifikant $(\mathrm{p}<.01)$. Die Autoren schlussfolgern, dass die positiven Ergebnisse aus Kurzinterventionsstudien in der ambulanten medizinischen Versorgung, die unter Forschungsbedingungen signifikante Unterschiede zwischen Interventions- und Kontrollbedingungen ermittelten, nicht ohne weiteres auf die Routineversorgung zu übertragen seien, und verweisen auf ähnliche Ergebnisse einer ebenfalls unter naturalistischen Bedingungen durchgeführten Studie von Richmond et al. [30].

Maisto et al. [26] führten in Syracuse (USA) ebenfalls in der ambulanten medizinischen Versorgung eine KI-Studie mit „riskanten“ und „leicht abhängigen Trinkern“ durch. 301 Patienten wurden nach einem positiven Screening (AUDIT und Häufigkeits-Menge-Index) randomisiert zwei Interventions- und einer Kontrollbedingung (Standardbehandlung) zugewiesen. Die erste Interventionsbedingung bestand in einem 10- bis 15-minütigen Gespräch, in dem den Patienten vor allem ein Feedback über die diagnostischen Befunde gegeben wurde. Die zweite Interventionsbedingung basierte auf dem im Project MATCH (s.o.) verwandten „Motivational Enhancement“ und sah insgesamt drei Gespräche von bis zu 45 Minuten Dauer vor. Die Interventionen wurden jeweils von speziell geschulten Fachleuten durchgeführt, die Standardbehandlung vom behandelnden Arzt. Nach 6 und auch nach 12 Monaten hatten die Patienten aller drei
Gruppen ihren Alkoholkonsum deutlich reduziert, ein signifikanter Unterschied zwischen den Interventionsgruppen und der Kontrollgruppe war allerdings bei keinem Messwert und zu keinem Messzeitpunkt auszumachen. Die Autoren erklären dies u. a. damit, dass sowohl im Hinblick auf die Kontrollgruppe die ausführliche Diagnostik als auch die aus ethischen Gründen in das Studiendesign aufgenommene Rückmeldung über die diagnostischen Befunde Interventionscharakter annahmen und somit keine „echte“ Kontrollbedingung vorlag. Auch sie verweisen auf andere KI-Studien, in denen Ähnliches beobachtet wurde.

Eine aktuelle Studie von Smith et al. [27] bestätigt die in der Übersichtsarbeit von Dunn et al. (ebd.) nachgewiesene Effektivität: 151 männliche Patienten einer gesichtschirurgischen Ambulanz eines Allgemeinkrankenhauses in Cardiff/Wales, die unmittelbar vor dem Unfall acht oder mehr Drinks konsumiert hatten, wurden randomisiert einer MI-Intervention oder einer Kontrollbedingung (keine alkoholspezifische Intervention) zugewiesen. Die Intervention bestand in einem einmaligen manualgestützten Gespräch (Dauer nicht mitgeteilt), das auf den wesentlichen Prinzipien und Strategien des MI (s.o.) aufbaute. Sowohl nach drei Monaten als auch nach einem Jahr wiesen die Patienten der Interventionsgruppe in allen konsumrelevanten Messwerten signifikant geringere Werte auf als die Patienten der Kontrollgruppe: Sie tranken zum einen deutlich weniger als zum Unfallzeitpunkt und zum anderen in den letzten drei Monaten vor dem Katamnesezeitpunkt deutlich weniger als die Patienten der Kontrollgruppe ( 3 Monate: $\mathrm{p}<.015$; 12 Monate: $\mathrm{p}<.031$ ). Nach einem Jahr hatte sich die Rate der riskant Konsumierenden in beiden Gruppen verringert, in der Interventionsgruppe jedoch in einem signifikant größeren Ausmaß ( $\mathrm{p}<.01)$.

Zusammenfassend liegen hier weitere Hinweise auf die Effektivität von KI auf MI-Basis gegenüber Kontrollen vor, es bleiben allerdings Fragen der differenzierten Wirksamkeit offen. Auch unterliegt der Übertrag von Forschungsdesigns und -ergebnissen in die Routineversorgung offenbar deutlichen Grenzen.

\section{Stand der Praxis in Deutschland}

Die praktische Umsetzung des MI-Kurzinterventionsansatzes in Deutschland lässt sich anhand der vorliegenden Literatur in drei Arbeitsfelder unterteilen: 


\section{Allgemeinkrankenhaus}

Die beiden großen vom Bundesministerium für Gesundheit geförderten Modellprojekte zu Kurzinterventionen bei Alkoholproblemen im Allgemeinkrankenhaus [11, 12] sowie ein weiteres großes multizentrisches Projekt des Frankfurter Instituts für Sozialarbeit und Sozialpädagogik [31] wiesen zwar positive Ergebnisse auf, eine Umsetzung in den Routinealltag nach Projekt- und damit Förderungsende konnte in allen Regionen jedoch nicht sichergestellt werden. Dies liegt vor allem daran, dass zur Sicherstellung eines systematischen Interventionskonzepts zusätzliche personelle Ressourcen nötig sind. Diese allerdings werden zurzeit weder von den einzelnen Krankenhäusern noch von den Krankenkassen - abgesehen von einigen wenigen Ausnahmen - zur Verfügung gestellt.

Einzelne Suchtberatungsstellen haben es sich teilweise schon seit mehreren Jahren zur Aufgabe gemacht, einzelne Krankenhäuser bzw. einzelne Abteilungen in Krankenhäusern ihres Einzugsgebiets aufzusuchen, um dort den Patientinnen und Patienten mit alkoholbezogenen Störungen - in aller Regel Alkoholabhängigkeit - eine gezielte Intervention anzubieten. So gelang es z. B. einer Suchtberatungsstelle im Berchtesgardener Land, mit drei Versorgungskrankenhäusern (dort den inneren Stationen) Kooperationsverträge abzuschließen, die einen regelmäßigen Liaison-Dienst zur Intervention bei alkoholbezogenen Störungen vorsehen [13]. Die kurzen Interventionen basieren auf dem Konzept des MI und haben das vorrangige Ziel, die Inanspruchnahme weiterführender Hilfen zu erhöhen, was nach einer mündlichen Mitteilung des verantwortlichen Sozialarbeiters auch in hohem Maße gelingt.

\section{Hausärztliche Praxen}

Nach Abschluss der Modellprojekte in Lübeck und Bielefeld konnte auch hier keine systematische Umsetzung von Kurzinterventionen der an den Projekten Beteiligten oder gar weiterer Praxen in den Routinealltag sichergestellt werden. Dies liegt vor allem daran, dass - so kurz sie auch sein mögen - alkoholspezifische qualifizierte KI Zeit und ein besonderes Maß an Aufmerksamkeit benötigen. Dies aber wird zumindest bislang im Vergleich mit anderen medizinischen Leistungen, die in derselben Zeit erbracht werden können, nicht ausreichend honoriert. Von einer systematischen Praxis MI-gestützter KI in Hausarztpraxen kann somit nicht die Rede sein.

\section{Fort- und Weiterbildungscurricula}

Hier sind drei Curricula zu nennen, in die das Wissen um MIKurzinterventionen Eingang gefunden hat:

- Suchtmedizinische Grundversorgung

Der Vorstand der Bundesärztekammer hat im September 1998 die sog. „Fachkunde Suchtmedizinische Grundversorgung“ beschlossen. Diese dient der „Vermittlung, dem Erwerb und dem Nachweis eingehender Kenntnisse, den Erfahrungen und den Fertigkeiten in der Prävention, Diagnostik, Therapie und Frührehabilitation von Suchterkrankungen“ [32, S. V]. Der Erwerb der Fachkunde ist prinzipiell Ärzten aller Gebietsbezeichnungen zugänglich - für substituierende Ärzte mittlerweile Pflicht - und setzt die Teilnahme an einem 50-stün- digen Weiterbildungskurs voraus. 12-14 Stunden dieser Weiterbildung dienen der Vermittlung von Kenntnissen über KI und dem Einüben einer praxisnahen motivierenden Gesprächsführung im Sinne des MI. Mittlerweile bieten alle Landesärztekammern derartige Weiterbildungen an. Die Nachfrage ist mäßig, was vor allem darauf zurückzuführen ist, dass der Erwerb der Fachkunde - neben der Ermächtigung zur Substitution Opiatabhängiger - keine erweiterten Abrechnungsmöglichkeiten erlaubt. Dennoch kann die Einführung der Fachkunde und mit ihr auch die Verankerung des (Brief) Motivational Interviewing in ärztlichen Weiterbildungskonzepten als versorgungspolitischer und konzeptioneller Fortschritt gewertet werden.

- Suchtpsychologische Grundversorgung

Die Deutsche Gesellschaft für Suchtpsychologie legte 2002 ein ebenfalls 50-stündiges Weiterbildungscurriculum „Fachkunde Suchtpsychologie“ für niedergelassene und angestellte Psychologen aus unterschiedlichen Tätigkeitsfeldern vor, in dem sie sich sehr eng an dem o.g. Curriculum der Bundesärztekammer orientierte. Auch hier nehmen MI und Kurzinterventionen einen breiten Raum ein (nachzulesen unter www.suchtpsychologie.de).

- MOVE - Motivierende Kurzintervention bei konsumierenden Jugendlichen

Das dritte Weiterbildungscurriculum schließlich richtet sich an Mitarbeiter der Jugendhilfe, die in direktem Kontakt mit konsumierenden Jugendlichen stehen und sich häufig hilfund sprachlos fühlen, wenn sie mit dem Konsum legaler oder illegaler Drogen konfrontiert sind oder von „ihren“ Jugendlichen dahingehend angesprochen werden. MOVE wurde im Auftrag des Landes NRW in Trägerschaft des ginko e.V. in Mülheim/Ruhr von einer interdisziplinär zusammengesetzten Arbeitsgruppe entwickelt, der u.a. auch der Autor angehörte (weitere Infos unter www.ginko-ev.de). Das Curriculum umfasst 24 Unterrichtsstunden. 10-12 davon dienen dem Erlernen konkreter Strategien der Gesprächsführung auf der Basis des MI. Der zentrale Stellenwert des MI in diesem Curriculum basiert auf der Erfahrung, dass MI am ehesten geeignet erscheint, mit den in der Regel hochambivalenten Einstellungen Jugendlicher gegenüber einer Einflussnahme Erwachsener konstruktiv umzugehen. Entwicklung und Erprobung des Curriculums werden von der Universität Bielefeld, Fakultät Gesundheitswissenschaften, wissenschaftlich begleitet. In den Jahren 2002 und 2003 wurden alle Suchtpräventionsfachkräfte Nordrhein-Westfalens als MOVE-Trainer ausgebildet, um entsprechende Kurse mit Mitarbeitern der Jugendhilfe in ihren Kommunen anzubieten.

Zusammenfassend liegen hier drei Weiterbildungscurricula vor, die sich Zielgruppen außerhalb der traditionellen Suchthilfe zuwenden und es somit in der praktischen Umsetzung mit konsumierenden Personen zu tun haben, die keine suchtspezifische Beratung oder Behandlung gesucht haben und gegenüber einer Veränderung eher ambivalent sind („non-treatment-seeking population“). Hier hat sich das Konzept KI auf Basis des MI schon in vielen Studien bewährt (s.o.). Folgerichtig wurde es in jeweils großem Umfang in die Curricula aufgenommen. 
In der Summe der hier berichteten Studienergebnisse können wir festhalten, dass hinreichend Evidenz für kurze Interventionen im Sinne des MI vorliegt. Die Evidenz bezieht sich sowohl auf Veränderungen von konsumbezogenen Verhaltensweisen als auch auf die Inanspruchnahme weiterführender Hilfen. Dies gilt im Grundsatz für Männer und Frauen.

In vielen Studien konnte jedoch die interne Validität des MI-Ansatzes (Manualtreue und Einhaltung des Untersuchungsprotokolls) nicht befriedigend bestätigt werden. Zwar wurden die für die KI verantwortlichen Therapeuten in allen Studien in der Anwendung MI-spezifischer Interventionen geschult, der Aufwand für die Trainings fiel allerdings sehr unterschiedlich aus, so dass von einer durchaus unterschiedlichen Qualität der KI ausgegangen werden muss. Darüber hinaus wurde in keiner Studie - nicht einmal stichprobenartig - die Umsetzung des Gelernten in die Praxis überprüft, wie es z.B. mittels Audio- oder Videoaufnahmen möglich wäre. Es bleibt deshalb unklar, inwieweit der MI-Ansatz konsequent verfolgt und somit als einzige Erklärung des Studienergebnisses gelten kann. Diese Einschränkung gilt allerdings für eine ganze Reihe von Interventionsstudien im Psychotherapiebereich. Den Studien zum MI wird in einer Übersichtsarbeit von Miller \& Wilbourne [33] trotz der beschriebenen methodischen Mängel eine im Vergleich mit anderen Untersuchungsansätzen im Suchtbereich gute methodologische Qualität zugesprochen. 17 Studien, die auf Basis des sog. „Motivational Enhancements“ durchgeführt wurden, erreichten einen durchschnittlichen Qualitätsindex von 13,12 und nahmen damit in einer Rangliste von 46 Behandlungsansätzen den zweiten Platz ein. $71 \%$ der hier berücksichtigten Studien wiesen positive Effekte auf.

Was aber sind die effektiven Komponenten der MI-gestützten KI? Burke et al. [34] fanden für die Wirksamkeit zweier Komponenten des MI deutliche Hinweise:

- empathischer Beratungsstil und

- sachliche Information/Rückmeldung über diagnostische Befunde.

Vergleicht man diese Komponenten mit den o.g. Themen kurzer MI-Interventionen [4], so stellt man eine deutliche Diskrepanz fest. Nicht die Inhalte der Interventionen scheinen nämlich in erster Linie für die Effektivität verantwortlich zu sein, sondern der Stil, die Haltung, die „Musik“ der Intervention: einfühlsam, nicht aggressiv, sachlich. Für differenziertere Aussagen reicht allerdings die momentane Datenlage nicht aus.

Einige der berichteten Studien weisen zum Nachbefragungszeitraum zwar signifikante Veränderungen der Konsumgewohnheiten auf, allerdings keine Unterschiede zwischen einzelnen Interventionsbedingungen oder gegenüber der Kontrollbedingung. Dies könnte ein Hinweis darauf sein, dass die in diesen Studien erreichten Zielgruppen (in der Regel nicht abhängig, keine schwerwiegenden Folgeprobleme) grundsätzlich von eher kurzen Interventionen profitieren. Eine detaillierte Voraussage allerdings, welche Zielgruppe am ehesten von MI-gestützten KI profitiert, lässt sich auf Basis der vorliegenden Literatur nur schwerlich vornehmen. Dazu sind die Ergebnisse zu uneindeutig.
Zusammengefasst ist weitere methodisch besser kontrollierte Forschung nötig, um die Effekte MI-gestützter KI differenziert zu ermitteln.

Was schließlich die Übertragbarkeit MI-gestützter KI in die Routineversorgung anbelangt, so ruht derzeit viel Hoffnung auf der ambulanten und stationären medizinischen Versorgung. In Münster (Hausarztpraxen; Arbeitsgruppe um F. Rist und R. Demmel), Greifswald (Allgemeinkrankenhaus: Arbeitsgruppe um U. Hapke) und Lübeck (Hausarztpraxen; Arbeitsgruppe um H.-J. Rumpf) werden zurzeit drei gut angelegte Studien zu MI-gestützten KI durchgeführt. Diese Projekte sollten den Bemühungen um eine Verankerung von alkoholspezifischen Kurzinterventionsansätzen in der medizinischen Versorgung zusätzlichen Antrieb verleihen. Dennoch ist hier Skepsis angezeigt. $\mathrm{Zu}$ einer systematischen Umsetzung von KI-Konzepten in der Routineversorgung muss es zunächst gelingen, die medizinische Versorgung für eine Verantwortungsübernahme zu gewinnen. Dazu gehören neben den grundsätzlichen konzeptionellen Entscheidungen im ambulanten Bereich eine Stärkung der „sprechenden Medizin“, im stationären Bereich vor allem zusätzliche personelle Ressourcen. An der mangelnden Evidenz MI-gestützter kurzer Beratungsansätze sollte eine Umsetzung nicht scheitern. Denn die ist - und das sollten die o. g. Studien gezeigt haben - gerade für die medizinische Versorgung hinreichend nachgewiesen.

\section{Literatur}

${ }^{1}$ Demmel R. Motivational Interviewing - ein Literaturüberblick. Sucht 2001; 47: $171-188$

2 Miller WR, Rollnick S. Motivational Interviewing - Preparing people to change addictive behavior. ( $\mathrm{dt}$. Übersetzung: Motivierende $\mathrm{Ge}-$ sprächsführung. Freiburg: Lambertus, 1999). New York: The Guilford Press, 1991

${ }^{3}$ Miller WR, Rollnick S. Motivational interviewing: Preparing people for change. New York: The Guilford Press, 2002

${ }^{4}$ Rollnick S, Heather N, Bell A. Negotiating behaviour change in medical settings: The development of brief motivational interviewing. Journal of Mental Health 1992; 1: 25-39

${ }^{5}$ Hester RK, Miller WR (eds). Handbook of alcoholism treatment approaches: Effective alternatives. Elmsford/NY: Pergamon Press, 1989

${ }^{6}$ Heather N, Rollnick S, Bell A et al. Effects of brief counselling among heavy drinkers identified on general hospital wards. Drug and Alcohol Review 1996; 15: 29 - 38

${ }^{7}$ Miller WR, Sanchez VC. Motivating young adults for treatment and lifestyle change. In: Howard G (Hrsg). Issues in alcohol use and misuse by young adults. Notre Dame: University of Notre Dame Press, 1991

${ }^{8}$ Babor TF. Avoiding the horrid and beastly sin of drunkenness: Does dissuation make a difference? Journal of Consulting and Clinical Psychology 1994; 62: $1127-1140$

${ }^{9}$ Moyer A, Finney JW, Swearingen CE et al. Brief interventions for alcohol problems: A meta-analytic review of controlled investigations in treatment-seeking and non-treatment-seeking populations. Addiction 2002; 97: 279-292

${ }^{10}$ Küfner H. Ergebnisse von Kurzinterventionen und Kurztherapien bei Alkoholismus - ein Überblick. Suchtmedizin 2000; 2: 181-192

${ }^{11}$ John U, Hapke U, Rumpf HJ et al. Prävalenz und Sekundärprävention von Alkoholmissbrauch und -abhängigkeit in der medizinischen Versorgung. Baden-Baden: Nomos, 1996

12 Kremer G, Dormann S, Wienberg G et al. Erkennung und Behandlung von PatientInnen mit Alkoholproblemen in der medizinischen Basisversorgung und Vernetzung mit dem Versorgungssystem für Abhängigkeitskranke. In: Bundesministerium für Gesundheit (Hrsg). Weiterentwicklung von Hilfen für Alkoholkranke und Menschen mit Alkoholproblemen. Baden-Baden: Nomos, 1998 
${ }^{13}$ FOGS GmbH. (Qualitäts-)Standards für Interventionen im Krankenhaus bei Menschen mit substanzbezogenen Störungen bzw. Risiken. Bericht der wissenschaftlichen Begleitung. Köln 2002

${ }^{14}$ Dunn C, DeRoo L, Rivara FP. The use of brief interventions adapted from motivational interviewing across behavioral domains: A systematic review. Addiction 2001; 96: 1725-1742

15 Bien TH, Miller WR, Boroughs JM. Motivational interviewing with alcohol outpatients. Behavioural and Cognitive Psychotherapy 1993; 23: $347-356$

${ }^{16}$ Miller WR, Benefield RG, Tonigan JS. Enhancing motivation for change in problem drinking: a controlled comparison of two therapist styles. Journal of Consulting and Clinical Psychology 1993; 61: 455-461

17 Project MATCH Research Group. Matching alcoholism treatments to client heterogeneity: Project MATCH posttreatment drinking outcomes. Journal of Studies on Alcohol 1997; 58: 7 - 29

18 Marlatt GA, Baer JS, Kivlahan DR et al. Screening and brief intervention for high-risk college student drinkers: results from a 2-year follow-up assessment. Journal of Consulting and Clinical Psychology 1998; 66: 604-615

${ }^{19}$ Handmaker NS, Miller WR, Manicke M. Findings of a pilot study of motivational interviewing with pregnant drinkers. Journal of Studies on Alcohol 1999; 60: 285-287

${ }^{20}$ Monti PM, Colby SM, Barnett NP et al. Brief intervention for harm reduction with alcohol-positive older adolescents in a hospital emergency department. Journal of Consulting and Clinical Psychology 1999; 67: 989-994

${ }^{21}$ Gentilello LM, Rivara FP, Donovan DM et al. Alcohol interventions in a trauma center as a means of reducing the risk of injury recurrence. Annals of Surgery 1999; 230: 473-483

${ }^{22}$ Schneider RJ, Casey J, Kohn R. Motivational versus confrontational interviewing: a comparison of substance abuse assessment practices at employee assistance programs. Journal of Behavioural Health Service Research 1999; 27: 60 - 74

${ }^{23}$ Miller WR. When is it motivational interviewing? Comment on Dunn et al's „The use of brief interventions adapted from motivational interviewing across behavioral domains: A systematic review“. Addiction 2001; 96: 1770-1772
${ }^{24}$ Senft RA, Polen MR, Freeborn DK et al. Brief intervention in a primary care setting for hazardous drinkers. American Journal of Preventive Medicine 1997; 13: 464-470

${ }^{25}$ Aalto M, Seppä K, Mattila P et al. Brief intervention for male heavy drinkers in routine general practice: a three year randomized controlled study. Alcohol \& Alcoholism 2001; 36: 224-230

${ }^{26}$ Maisto SA, Conigliaro J, McNeil M et al. Effects of two types of brief intervention and readiness to change on alcohol use in hazardous drinkers. Journal of Studies on Alcohol 2001; 62: 605-614

27 Smith AJ, Hodgson RJ, Bridgeman K et al. A randomised controlled trial of a brief intervention after alcohol-related facial injury. Addiction 2003; 98: $43-52$

28 Babor TF, De La Fuente JR, Saunders J et al. AUDIT: The Alcohol Use Disorders Identification Test: Guidelines for use in primary health care. WHO Publications No. 89.4. Geneva: World Health Organization, 1989

${ }^{29}$ Mayfield D, McLeod G, Hall P. The CAGE questionnaire: validation of a new alcoholism screening instrument. American Journal of Psychiatry 1974; $131: 1121-1123$

${ }^{30}$ Richmond R, Heather N, Wodak A et al. Controlled evaluation of a general practice-based brief intervention for excessive drinking. Addiction 1995; 90: 119-132

${ }^{31}$ Arnold T, Schmid M, Simmedinger R. Suchthilfe im Krankenhaus. Baden-Baden: Nomos, 1999

32 Bundesärztekammer (Hrsg). Curriculum Suchtmedizinische Grundversorgung. Texte und Materialien der Bundesärztekammer zur Fortbildung und Weiterbildung, Bd. 20. Köln 1999

${ }^{33}$ Miller WR, Wilbourne PL. A methodological analysis of clinical trials of treatments for alcohol use disorders. Addiction 2002; 97: 265 - 277

34 Burke BL, Arkowitz H, Dunn C. The Efficacy of Motivational Interviewing and its adaptations - What we know so far. In: Miller WR, Rollnick S (Hrsg). Motivational interviewing: Preparing people for change. New York: The Guilford Press, 2002 